\title{
Reliability Analysis and Optimization of the Ship Ballast Water System
}

\author{
Tang Ming ${ }^{1}$, Zhu Fa-xin ${ }^{2, *}$ and Li Yu-le ${ }^{2}$ \\ ${ }^{1}$ JiangSu Maritime Institute, NanJing, P. R. China \\ ${ }^{2}$ Maritime College of ZheJiang Ocean University, ZhouShan, P. R. China
}

\begin{abstract}
In this paper, the ship ballast water system was considered as an object of study, and the problem to be solved is to improve its reliability. The reliability of the ship ballast water system is improved to a higher level, as it improves the ship's safety, because it decides about the stability, running resistance and also the cargo's safety on the ship. The transportation failure of the ballast water in the ship ballast water system was considered as the top event, thus, a fault tree was established according to the fuzzy set theory, and then the reliability calculation was carried out. Furthermore, the ballast water system was optimized according to the frequent faults occurring in the past, its reliability is calculated again in the same way. The calculated results showed that the optimized ballast water system has a higher reliability value. The core innovation in the study is to solve the problem that involves the uncertainty of randomness and fuzziness by applying a fuzzy number into the fault tree analysis. From the example of reliability analysis of the ship ballast water system, the proposed method of the application of the fuzzy fault tree theory in reliability analysis is verified effectively.
\end{abstract}

Keywords: Fuzzy fault tree, Optimal design, Reliability, The ship ballast water system.

\section{INTRODUCTION}

Main function of the ship ballast water system is to maintain a proper displacement, water line, longitudinal and transverse balance of the ship hull by transporting seawater into the ballast water tanks or discharging ballast water outboard. So the safety of the ship will be affected directly if the ballast water system has some faults. In this paper, the fuzzy fault tree of the ballast water system was set up, its reliability was calculated and analyzed by the means of the fuzzy algorithms.

\section{THE FUZZY FAULT TREE ANALYSIS}

\subsection{Fault Tree Analysis}

Fault tree analysis is a very important method used in the system reliability study. The basic idea of the method is that the failure of system is regarded as the top event and more information is obtained in the same way [1]. Firstly, a person should ask the question: "what are the direct factors that could cause the top event?" Then list the factors, like A, B, C and so on. Secondly, the person chooses direct factors listed above and does some further research to figure out what are the causing factors behind the direct ones. In accordance with the step by step approach, the researcher traces back to the most basic events. With the quantitative calculation of the traditional fault tree analysis, you need to know the exact value of the probability of the end events. However, it is

*Address correspondence to this author at the No.1, Haida South Road, Lincheng Changzhi Island, Zhoushan, Zhejiang, P.R. China. Postcard: 316022; Tel: 15858050245; E-mail: zhufax7758@163.com hard to obtain the exact value of the end events in many cases. So a fuzzy number is commonly introduced to make up this shortfall for the traditional fault tree in engineering fields $[2,3]$.

\subsection{The Introduction of the Fuzzy Numbers}

Applying fuzzy number into the fault tree analysis can solve the problem that involves the uncertainty of randomness and fuzziness. If the probability of a certain event is between 0.2 and 0.3 , such as it may be 0.28 , then we can

define it as a fuzzy number [4]. Normally $\tilde{p}=(\mathrm{m}, \alpha, \beta)$, in this equation, $m$ is the average value of $\tilde{p}, \alpha, \beta$ are the distribution parameters on both sides. If $\alpha, \beta$ are both zero, then $p$ is a fuzzy number.

The rough membership function of $\tilde{p}$ satisfies:

$u_{A(x)}=\left\{\begin{array}{cc}L\left(\frac{m-x}{\alpha}\right) & x \leq m, \alpha>0 \\ R\left(\frac{x-m}{\beta}\right) & x>m, \alpha>0\end{array}\right.$

So $p$ is the fuzzy number of L-R. There are many functions of this kind in the engineering field, such as triangle function, normal function and the trapezoidal function as shown in Fig. (1). 


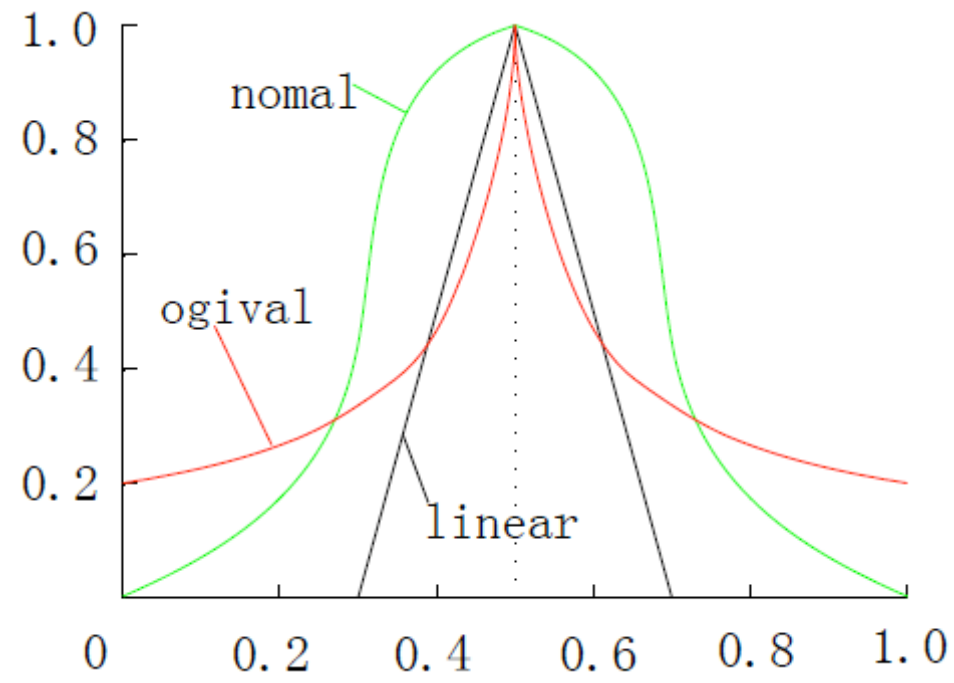

Fig. (1). Three forms of common membership functions.

The statistical method is commonly used to accurately determine the membership function. For the linear membership function, fuzzy numbers can be expressed as:

$p=(m-\alpha, m, m+\beta)$

If $a=m-\alpha$

and $b=m+\beta$

Fuzzy number can be expressed as $p=(a, m, b)$. By using a fuzzy number to replace the accurate value of the probability with the practical experience and judgment of engineering technicians, the membership function of fuzzy number can be established. An error can be tolerated to a certain extent, with greater flexibility and adaptability [5].

\subsection{Fuzzy Algorithms}

If we say $\bar{p}_{1}=\left(a_{1}, m_{1}, b_{1}\right)$ and $\bar{p}_{2}=\left(a_{2}, m_{2}, b_{2}\right)$, then the algorithms can be described as in the following equations :

The addition rule:

$\tilde{p_{1}} \tilde{+} \tilde{p}_{2}=\left(a_{1}, m_{1}, b_{1}\right) \tilde{+}\left(a_{2}, m_{2}, b_{2}\right)=\left(a_{1}+a_{2}, m_{1}+m_{2}, b_{1}+b_{2}\right)$

The subtraction rule:

$\tilde{p_{1}} \tilde{-} \tilde{p}_{2}=\left(a_{1}, m_{1}, b_{1}\right) \tilde{-}\left(a_{2}, m_{2}, b_{2}\right)=\left(a_{1}-a_{2}, m_{1}-m_{2}, b_{1}-b_{2}\right)$

The multiplication rule:

$\tilde{p_{1}} \tilde{\times} p_{2}=\left(a_{1}, m_{1}, b_{1}\right) \tilde{\times}\left(a_{2}, m_{2}, b_{2}\right)=\left(a_{1} a_{2}, m_{1} m_{2}, b_{1} b_{2}\right)$

The division rule:

$\tilde{p_{1}} \div \tilde{p}_{1}=\left(a_{1}, m_{1}, b_{1}\right) \div\left(a_{2}, m_{2}, b_{2}\right)=\left[\frac{a_{1}}{b_{2}}, \frac{m_{1}}{m_{2}}, \frac{b_{1}}{a_{2}}\right]$
It should be noted that the above fuzzy number multiplication and division formulas are established approximately.

\section{RELIABILITY CALCULATION OF THE SHIP BALLAST WATER SYSTEM}

\subsection{The Working Principle of the Ship Ballast Water System}

Fig. (2) shows the working principle of ballast water system on a board ship. There are five ballast water tanks located in the bow, stern, port and starboard of the ship. All the ballast water manifolds from each water ballast tank are gathered together in the valve box 3 . The inlet and outlet manifolds of the valve box 3 are connected to the discharge and suction ports of the total pump 2 and fire pump 1 is on standby.

In addition, suction ports of two pumps can be connected to the seawater manifold by the use of the gate valve. The discharge ports of two Pumps are also connected to nonreturn valves on both sides with pipework. When transporting seawater to the tanks, total pump 2 (or the fire pump 1) can be used to draw water through the seawater door outboard, then pump the seawater via discharge ports to the required tanks by using the valve box. When discharging seawater outboard, the total pump 2 (or the fire pump 1) is used to draw from some tanks, and pump it outboard by discharge valves on the sides.

\subsection{Fault Tree of the Ship Ballast Water System}

Fig. (3) shows the fault tree which takes the transportation failure of ballast water system as the top event. Besides the top event, there are also middle events and bottom events. The middle events are some faults which can cause the top event and continue to be divided into the next stage events, and the bottom events cannot be divided further. All kinds of events are shown in Table $\mathbf{1}$ below. 


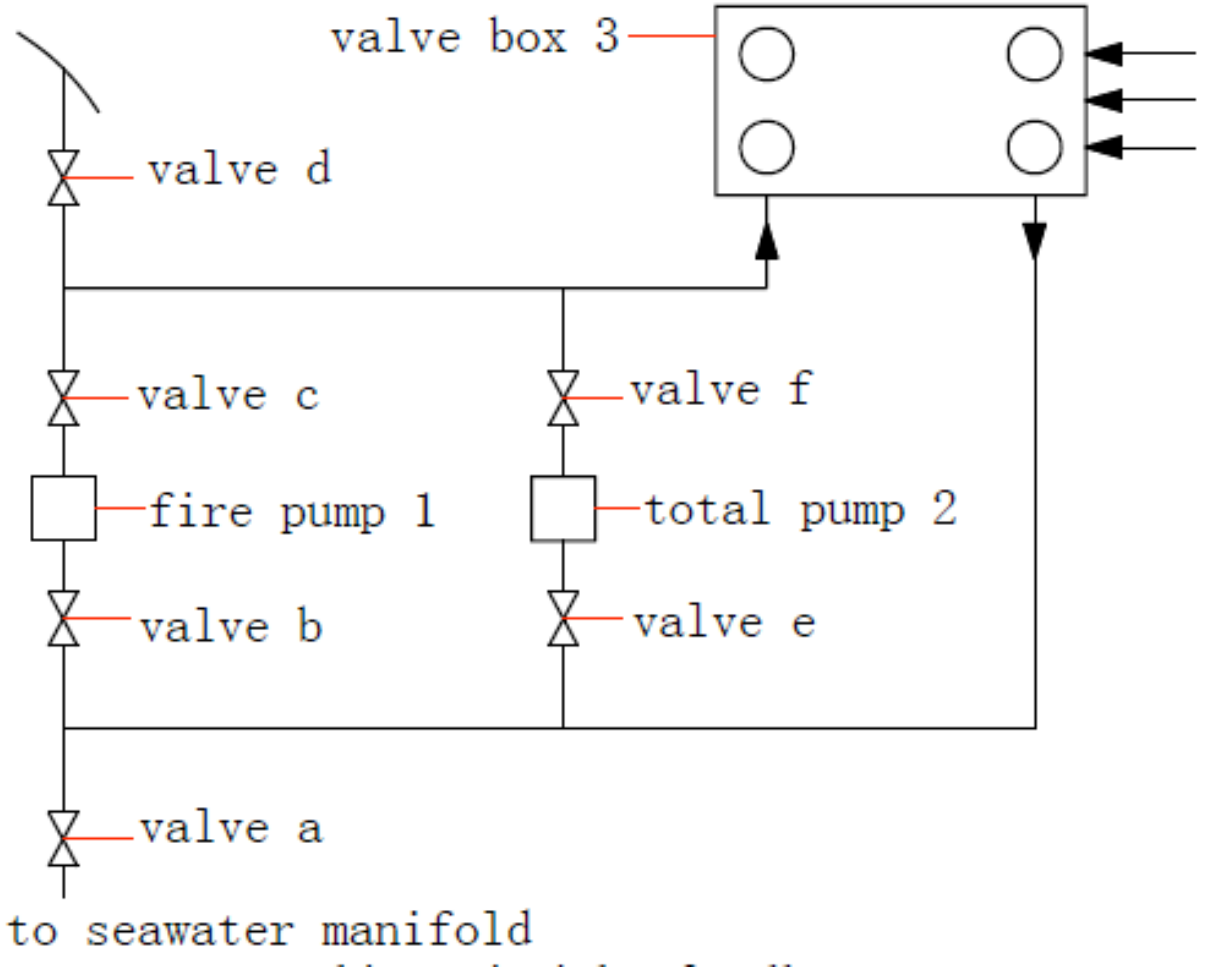

崩

Fig. (2). Working principle of ballast water system schematic.

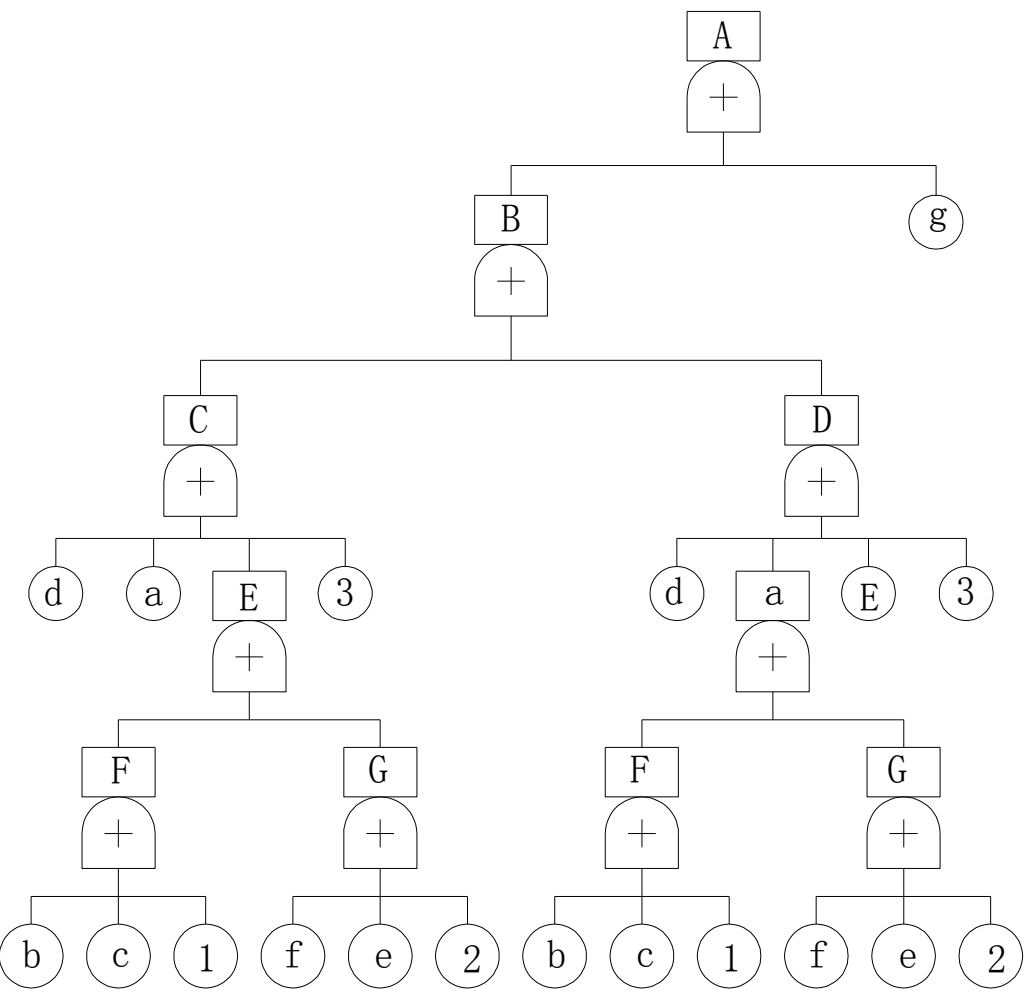

Fig. (3). Fault tree of the ballast water system. 
Table 1. The fuzzy number of every event.

\begin{tabular}{|c|c|c|c|}
\hline Event & Name of Event & Average Value & $\alpha$ and $\beta$ \\
\hline A & Failure of V/V.a & 0.02020 & 0.00898 \\
\hline $\mathrm{c}$ & Failure of V/V.c & 0.02020 & 0.00898 \\
\hline e & Failure of V/V.e & 0.02020 & 0.00898 \\
\hline $\mathrm{f}$ & Failure of V/V.f & 0.02020 & 0.00898 \\
\hline $\mathrm{g}$ & Pipelines Broken & 0.00003 & 0.00001 \\
\hline 1 & Fire Pump Malfunction & 0.00675 & 0.00300 \\
\hline $\mathrm{B}$ & Failure of Suction(S) and Discharge (D) & 0.00196 & 0.00087 \\
\hline $\mathrm{C}$ & Failure of S & 0.04427 & 0.01968 \\
\hline $\mathrm{D}$ & Failure of D & 0.04427 & 0.01968 \\
\hline E & Failure of Two Pumps & 0.00246 & 0.00109 \\
\hline $\mathrm{F}$ & Failure of Fire Pump & 0.04647 & 0.02065 \\
\hline G & Failure of Total Pump & 0.05295 & 0.02353 \\
\hline
\end{tabular}

\subsection{The Fuzzy Equations of Fault Tree Analysis}

Based on the fuzzy algorithms of the fuzzy number, Fuzzy equations of the fault tree analysis are expressed as follows, if the probability of occurrence of the event $i$ is a fuzzy number $P_{i}$, the fuzzy equation of AND gate can be given as:

$$
P_{A N D}=A N D\left(\underset{\sim}{\sim} \underset{P_{1} P_{2} \cdots \cdots}{\sim} P_{n}\right)=\prod_{i=1}^{n} \sim p_{i}
$$

the fuzzy equation of OR gate is given as:

$$
P_{O R}=\operatorname{OR}\left(\underset{P_{1} P_{2}}{\sim} \cdots \cdots P_{n}\right)=1-\prod_{i=1}^{n} 1-\stackrel{\sim}{\sim}
$$

\subsection{Reliability Calculation of the Ship Ballast Water Sys-} tem

Assuming that the basic events are independent of each other, then the truth-function of the top event in Fig. (3) is as follows :

$$
\underset{\sim}{P_{A}}=\stackrel{\sim}{\sim} p_{B}+p_{g}
$$

$$
\begin{aligned}
& \sim \sim \sim \\
& P_{B}=p_{C} \cdot p_{D} \\
& P_{E}=p_{F} \cdot p_{G} \\
& P_{F}=p_{b}+p_{c}+p_{1} \\
& P_{G}=p_{f}+p_{e}+p_{2}
\end{aligned}
$$$$
\sim \sim \sim \sim \sim \sim \sim \sim \sim \sim
$$$$
P_{C}=p_{a}+p_{d}+p_{3}+p_{E} P_{\underset{D}{D}}=p_{a}+p_{d}+p_{3}+p_{E}
$$

In order to calculate easily, the triangle membership function shown in Fig. (1) is chosen as the membership function, which can be expressed as:

$\mathcal{U}_{A(x)}=\left\{\begin{array}{cc}0 & x<a \\ (x-a) /(m-a) & a \leq x<m \\ (b-x) /(b-m) & m \leq x \leq b \\ 0 & x>b\end{array}\right.$ 

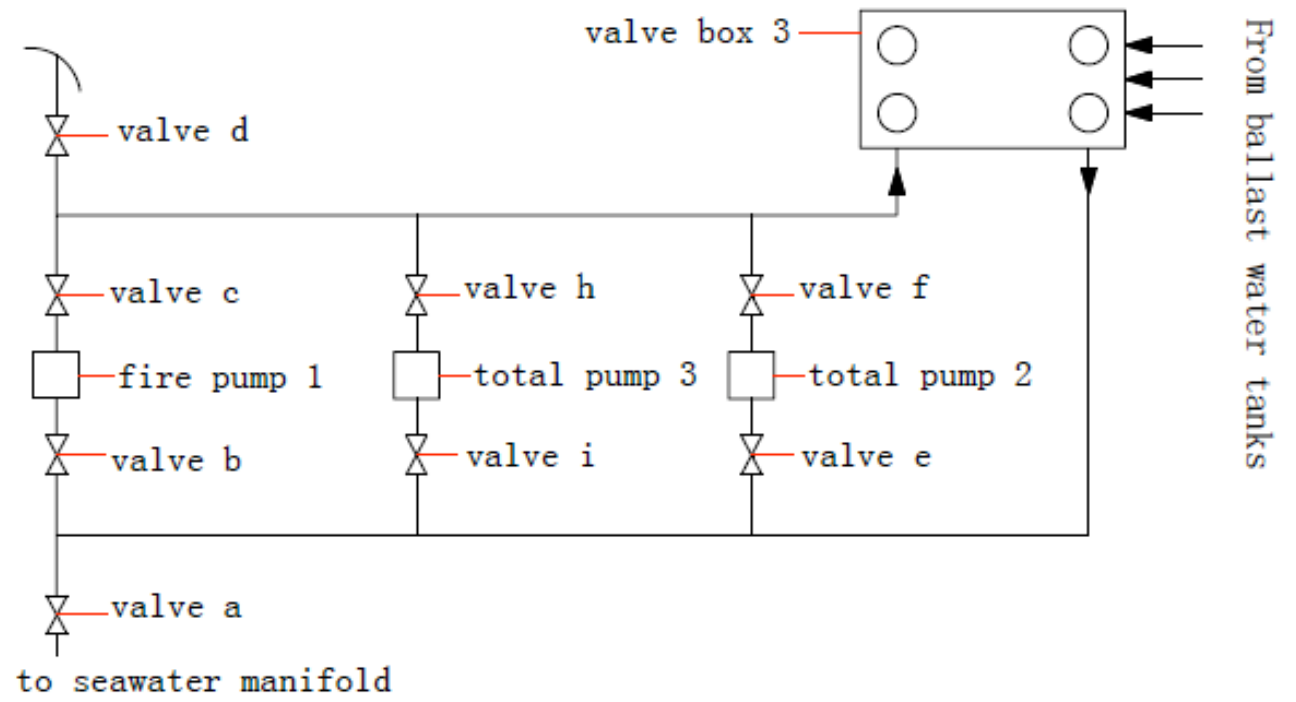

Fig. (4). The optimized ballast water system.

Presuming that the degree of membership for point $\mathrm{x}$, which has a $60 \%$ difference from the average value, is 0.1 , then $(0.6 m-a) /(m-a)=0.1$ and $a=5 m / 9$ are given, $\alpha$ and $\beta$ of the basic event are calculated readily with $a=m-\alpha$ and $b=m+\beta$ according to the table. The fuzzy number of each middle event and the top event are calculated according to the algorithm of the fuzzy number and the fuzzy equations. The fuzzy number of every event in the fault tree is shown in Table $\mathbf{1}$.

If the traditional fault tree analysis is used, the probability of the top event calculated is a certain value of 0.00199 , while the fuzzy number calculation is adopted, the probability of the top event is as follows:

$$
\underset{\sim}{P_{A}}=(0.00060,0.00199,0.00423) .
$$

The result can better reflect the ambiguity of the event, achieving a more realistic situation because ship ballast water plays an important role in controlling the stability of the ship, it is necessary to further improve the reliability of the ballast water system.

\section{OPTIMIZATION OF THE SHIP BALLAST WATER SYSTEM}

According to the previous statistical data of the faults in the ship ballast water system, the fault happened most frequently in the water pump or its pipework. As this fault might most likely occurred in the water pump or its pipework, the ballast water system was optimized by connecting in parallel to another fire pump and its pipework beside the fire pump. Now there are two fire pumps on standby in the ballast water system, and if a fault occurs in one fire pump, another will come into use immediately, so the reliability of the ballast water system is improved to a higher value. The optimized ballast water system is shown in Fig. (4).

Again, the transportation failure of the ballast water in the optimized ballast water system was taken as the top event and fault tree relevance was also established. The reliability value of the optimized ballast water system was calculated by the fuzzy algorithms in the fuzzy set theory, and the fuzzy probability of optimized ballast water system is expressed as follows.

$$
\underset{\sim}{P_{A}}=(0.00057,0.00180,0.00371) .
$$

\section{RESULTS}

According to calculated results of the former ballast water system and the optimized ballast water system, the reliability value of the optimized ballast water system was improved by $9.5 \%$ as compared to the former one.

\section{CONCLUSION}

According to the example of the application of fuzzy fault tree theory in the reliability analysis of the ship ballast water system, it was easily concluded that the fuzzy fault tree theory could solve the problem as the traditional fault tree analysis had difficulty in obtaining the precise probabilities of the basic events. The reliability of the ballast water system was improved reasonably to a higher value by the optimal design. The methods of fuzzy fault tree theory are applied to the reliability analysis and the optimization of other systems is effectively achieved.

\section{CONFLICT OF INTEREST}

The authors confirm that this article content has no conflict of interest. 


\section{ACKNOWLEDGEMENTS}

The present research was partly funded by Zhejiang Provincial Natural Science Foundation of China, No. LQ14E090001. Also, the research was supported by ZheJiang Ocean University and JiangSu Maritime Institute. Last, thanks to Zhang Di, Lei Jian and other people because of your help sincerely.

\section{REFERENCES}

[1] Y. Dong, W. Zhu, and X. Chen, "The analysis and application of fuzzy number", Hefei Univ. Technol. (Nat. Sci. ed.), vol. 19, no. 04, pp. 35-41, Dec.1996. (in Chinese)
[2] C. Yang, and Q. Zhang, "The fuzzy design and evaluation in hydraulic system reliability", Construction Machinery, vol. 23, no. 12, pp. 61-64, Dec. 2003. (in Chinese)

[3] L. Zhao, Y. Ji, "Fault tree analysis of the hydraulic system fault diagnosis", Mach. Tool Hydraul., vol. 37, no. 02, pp. 199-200, Feb. 2009. (in Chinese)

[4] C. Hou, Z. Yuan, and C. He, "Electro-hydraulic steering gear reliability based on fuzzy fault tree analysis", Mach. Tool Hydraul., vol. 35, no. 06, pp. 226-227, Jun. 2007. (in Chinese)

[5] G. Liu, S. Lin, X. Jiang, Q. Chen, and S. Liu, "Reliability of the ship winch hydraulic system simulation based on fault tree analysis", Ship \& Ocean Eng., vol. 37, no. 02, pp. 70-73, 2008. (in Chinese)

(C) Ming et al.; Licensee Bentham Open.

This is an open access article licensed under the terms of the Creative Commons Attribution Non-Commercial License (http://creativecommons.org/licenses/ by-nc/4.0/) which permits unrestricted, non-commercial use, distribution and reproduction in any medium, provided the work is properly cited. 